\title{
Das Gesellschaftliche als Aporie - Plädoyer für eine poststrukturalistisch informierte Diskurs-Praktiken-Forschung im Kontext der Transformation Sozialer Arbeit
}

\author{
Marek Naumann $\cdot$ Nils Wenzler $\cdot$ Yasmine Chehata $\cdot$ Udo Seelmeyer
}

Online publiziert: 8. Juni 2017

(C) Der/die Autor(en) 2017. Dieser Artikel ist eine Open-Access-Publikation.

Zusammenfassung Der vorliegende Beitrag fragt nach dem analytischen Mehrwert poststrukturalistischer Forschungszugänge im Kontext einer Transformation Sozialer Arbeit. Ausgehend von der Diagnose des sozialstaatlichen Wandels im Sinne einer Neujustierung des wohlfahrtsstaatlichen Arrangements, wird dieses in ein poststrukturalistisches Theorieverständnis eingebettet und auf die Frage möglicher Lesarten von Diskurs-Praktiken-Verhältnissen zugespitzt. Vor dem Hintergrund von vier sodann vorgestellten Konturierungsversuchen, gründend auf unterschiedlichen Forschungs- bzw. Qualifikationsprojekten, wird für die Aufgabe eines allzu starren Mikro-Makro-Dualismus plädiert und die Öffnung hin zu Forschungsdesigns angeregt, die empirisch an die Idee der Gouvernementalitätsanalytik anknüpfen.

Schlüsselwörter Transformationsforschung · Methodologie · Praktiken · Diskurse $\cdot$ Machtanalytik

\footnotetext{
Dipl.-Päd. M. Naumann ( $\bowtie)$

Institut für regionale Innovation und Sozialforschung e. V., Räcknitzhöhe 35a, 01217 Dresden, Deutschland

E-Mail: naumann@iris-ev.de
}

Dipl.-Päd. N. Wenzler, M.A.

Fakultät für angewandte Sozialwissenschaften, Technische Hochschule Köln,

Gustav-Heinemann-Ufer 54, 50968 Köln, Deutschland

E-Mail: nils.wenzler@th-koeln.de

Dipl. Soz.arb. Y. Chehata, M.A.

Fakultät für angewandte Sozialwissenschaften, Institut für Kindheit, Jugend, Familie und Erwachsene, Technische Hochschule Köln, Gustav-Heinemann-Ufer 54, 50968 Köln, Deutschland E-Mail: yasmine.chehata@th-koeln.de

Prof. Dr. U. Seelmeyer

Fakultät für angewandte Sozialwissenschaften, Institut für die Wissenschaft der Sozialen Arbeit, Technische Hochschule Köln, Ubierring 48, 50678 Köln, Deutschland

E-Mail: udo.seelmeyer@th-koeln.de 


\title{
The Social as aporia - Plea for a poststructuralist informed discourse- practice-research in the context of transformation of social work
}

\begin{abstract}
The present contribution investigates the added value of post-structuralist research approaches in the context of a transformation of social work. Based on a diagnosis which sees an ongoing change of the social state towards readjusting the arrangements of its social-welfare properties, a post-structuralist understanding of theory is used and focused on the question of potential interpretations of discourse-practices-relationships. Four approaches to outline this in more detail are offered, informed by different research and qualification projects. On this backdrop, the authors strongly suggest to refrain from employing a too rigid micro-macrodualism in favour of open research designs, which empirically tie in with the idea of governmentality analytics.
\end{abstract}

Keywords Research on transformation - Methodology · Practices · Discourse · Analytics of governmentality

\section{Vom Wohlfahrtsstaat zum postwohlfahrtsstaatlichen Arrangement}

Seit den 1970er Jahren befindet sich der bundesrepublikanische Wohlfahrtsstaat in einem sichtbaren Wandel. Während dieser sich zu Beginn und bis hinein in die 1990er Jahre eher gemächlich vollzog, lassen sich in den letzten zwanzig Jahren tiefgreifende Veränderungen beobachten. Auch die Soziale Arbeit ist von diesem Transformationsgeschehen sichtbar berührt: Gleichwohl erste Versuche einer entsprechenden Dechiffrierung dieses Verhältnisses noch unter dem Eindruck einer scheinbaren Indienstnahme für den ,aktivierenden Sozialstaat“ (Dahme et al. 2008, 2003a) standen, manifestierte sich wenig später die Einsicht, dass eine ,aktivierende Soziale Arbeit“ (Dahme und Wohlfahrt 2005) bzw. ,Sozialpädagogik“ (Dollinger und Raithel 2006) bedeutsame Akteur*in bzw. ,aktivierungspädagogischer Transmissionsriemen“ (Kessl 2005) innerhalb des Umbauprozesses ist. Nicht zuletzt vor diesem Hintergrund wurde auch im Rahmen des Forschungsprogramms des Promotionskollegs „Leben im transformierten Sozialstaat“ die Überzeugung zugrunde gelegt, dass zwischen Sozialpolitik, als gesellschaftsregulierender Praxis des Wohlfahrtsstaates, und Sozialer Arbeit ein unmittelbarer Wirkungszusammenhang besteht.

Innerhalb der sozialpädagogischen Debatten lässt sich eine starke Position dahingehend ausmachen, dass Soziale Arbeit, als sozialstaatliche Dienstleistung verstanden, die neuen gesellschaftlichen Normen der Eigenverantwortung, Autonomie und Aktivierung, als Kernparadigmen des gegenwärtigen sozialstaatlichen Wandels, „programmgemäß und standortorientiert“ (Dahme et al. 2003b, S. 11) um- bzw. durchsetzt. Diese besondere Beziehung zwischen Sozialstaat und Sozialer Arbeit gründe, so die Argumentation, auf einem gewachsenen Verhältnis, insofern Soziale Arbeit vor allem seit den 1970er Jahren organisatorisch, programmatisch wie nicht zuletzt auch strategisch in die sozialpolitischen Ordnungszusammenhänge eingebunden wurde (Dahme und Wohlfahrt 2008). Eine solche Lesart im Sinne eines 
direktiven Wirkungszusammenhangs zwischen sozialpolitischer Zielintention und sozialarbeiterischer/sozialpädagogischer Durchsetzungspraxis hat zweifelsohne eine gewisse Plausibilität, allerdings erzeugt sie - erkenntnistheoretisch gesehen - ein eher ernüchterndes und unbefriedigendes Bild. Denn entweder wird Soziale Arbeit auf diese Weise als handlungsohnmächtige bzw. willfährige „Durchlauferhitzerin“ sozialstaatlicher Neuprogrammierung konturiert oder aber - im Sinne einer kritischen Sozialen Arbeit - als ,heroische Widerstandskämpferin“ gegen neosoziale Verhältnisse aufgerufen. Bei aller Diversität in der Argumentation teilen beide Interpretationsangebote die Vorstellung vom (Sozial-)Staat als Überbauphänomen.

Hiergegen ließe sich - in einer ersten Absetzbewegung - mit Stephan Lessenich (2012) die Einsicht mobilisieren, dass der Sozialstaat keineswegs nur treibender, sondern durchaus auch getriebener Akteur im Rahmen gesellschaftlicher (Um-)Strukturierungsprozesse ist, dass er damit in gleicher Weise strukturierter wie strukturierender Natur ist. Treibt man dieses Monitum gegen die scheinbare Allmacht des Staates noch ein wenig weiter, kann dieser mit Nicos Poulantzas (2002) auch als ,Verdichtung eines Kräfteverhältnisses“ (Poulantzas 2002, S. 114) verstanden werden. Ein solcher Relativismus gestattet es, vom Staat als priorisiertem Ausgangspunkt wohlfahrtsstaatlicher Transformationsdiagnosen abzurücken und den Fokus deutlicher auf die Frage gesellschaftlicher Machtverhältnisse, Kämpfe und Aushandlungsprozesse zu lenken. Damit wird keineswegs die praktische wie analytische Relevanz staatlich-institutioneller Zusammenhänge bestritten, allerdings nach der Spezifik ihrer Materialisierung und den wohlfahrtsstaatlichen Ordnungen wie paradigmatischer Handlungsweisen in gesellschaftlichen Macht- und Herrschaftsbeziehungen gefragt. An die Stelle einer unhinterfragten „Ontologisierung des Staates“ (Foucault 2003a, S. 791), wie sie gewissermaßen den Überbau- und Strukturrhetoriken zugrunde liegt, lässt sich auf diese Weise die komplexe und unterschiedlich akzentuierbare Frage nach der „Ökonomie der Machtbeziehungen“ (Foucault 2003b, S. 677, 2003a) rücken.

Vor diesem Hintergrund schließen wir an Überlegungen von Fabian Kessl und Hans-Uwe Otto (2008) ebenso wie an die von Holger Ziegler (2008) an, die vom wohlfahrtsstaatlichen bzw. sozialstaatlichen Arrangement sprechen, um so die Aufmerksamkeit vom Staat selbst auf die spezifischen Denk-, Handlungs- wie auch Problematisierungsweisen zu lenken, welche den Rahmen gesellschaftlicher und damit auch staatlicher Rationalitäten abgeben. Das Arrangement ist dabei nicht als ein fixiertes Korsett zu verstehen, sondern als Heuristik, um Macht-Wissen-Ordnungen begreifbar und analytisch zugänglich zu machen. In dieser Weise lässt sich nun das (vertraute) wohlfahrtsstaatliche Arrangement als historisch kontingenter Rationalisierungsmodus verstehen, der die staatlich organisierte Kollektivierung gesellschaftlicher Risikobearbeitung in den Mittelpunkt stellte. Im fortgeschrittenen 19. Jahrhundert hatte sich diese im Zuge einer Problematisierung liberaler Regierungsweisen konstituiert. Die damit einhergehende „Erfindung des Sozialen“ profilierte Denkund Handlungsweisen, die das Regierungswissen und -handeln in den Zusammenhang einer kollektivierten Existenzweise von Bevölkerung als „Gesellschaft“ stellte (vgl. etwa Donzelot 1994). Die sich dann abzeichnende Anrufung und Indienstnahme des Staates als (nationalstaatliches) Organisationsprinzip für das Soziale lässt sich sodann als eine Rationalisierungspraxis beschreiben, in deren Gefolge sich ein 
„,sozialer Sektor“ zu materialisieren begann. Dieser wurde zwar in Abgrenzung zu den unmittelbaren „kapitalistischen Verwertungszusammenhängen“ unter der Ägide einer eigenen Wissensordnung entworfen, allerdings blieb die Frage der Risikoproduktion kapitalistischer Vergesellschaftung permanenter Bezugspunkt der Entwicklung. Die Soziale Arbeit ist vor diesem Hintergrund nun keineswegs und zuerst als absichtsvolle Einrichtung des Sozialstaates aufzufassen, sondern muss - mit Bezug auf die Idee kollektiver Risikobearbeitung - als sich tendenziell eigenständig formierende Macht-Wissen-Ordnung verstanden werden. Während der Sozialstaat im Sinne des Sozialversicherungssystems vor allem eine biopolitische, überdies auch disziplinarische Regulierungsfunktion übernahm, organisierte sich Soziale Arbeit als eine Wissens- und Handlungsformation, die, gleichsam in diesem Problemkontext verortet, auf problematisch markierte Lebensführungsweisen reflektierte (Kessl und Otto 2012; Ziegler 2008).

Die Krise des Wohlfahrtsstaates, wie wir sie seit den 1970er Jahren erleben, lässt sich auch als Problematisierung dieses wohlfahrtsstaatlichen Arrangements beschreiben. Deutlich zu kurz greifen vor diesem Hintergrund Argumentationen, die dessen Kern in einem sozialstaatlichen Abbau bzw. dem Abschmelzen der sozialen Idee überhaupt wähnen. Vielmehr zeichnet sich zum einen ab, dass nun der eigenverantwortliche, autonome und aktive Bürger deutlicher adressiert wird, Teil der (Re-)Produktion des Sozialen zu sein, und zum anderen, dass - hierzu ergänzend der Sozialstaat seine Ordnungspraxis neu codiert, von der Idee der Unmittelbarkeit hin zur Maxime eines „Regierens auf Distanz“ (Miller und Rose 1994).

Das post-wohlfahrtsstaatliche Arrangement, als ein heuristischer Zugriff auf die theoretische Erfassbarkeit einer „Neuerfindung des Sozialen“ (Lessenich 2008), kann nun aber keineswegs - wie vielmals geschehen - allein unter der Perspektive einer „Ökonomisierung des Sozialen“ betrachtet und weniger noch auf eine allgegenwärtige Subjektfigur des „Unternehmers seiner Selbst“ (Bröckling 2007) hin verhandelt werden. Die Problematisierungs- und Bearbeitungsweisen innerhalb des postwohlfahrtsstaatlichen Arrangements sind, je nach in Blick genommenem Kontext, uneinheitlich und vielschichtig zugleich. Dies macht es zum einen schwierig, die Transformation von Sozialstaatlichkeit signifikant zu erfassen bzw. zu bestimmen, andererseits öffnet es den Blick für die komplexen Machtspiele im Kontext der Neujustierung sozialer Institutionalisierungsweisen. Denn diese sind eben nicht als einheitliche Bewegung zu kennzeichnen, sondern verweisen auf Kämpfe „zwischen individuellen und kollektiven Interessen, zwischen dem Gleichgewicht des Marktes und der Herrschaft der öffentlichen Gewalt" (Foucault zit. nach Kessl und Otto 2008, S. 9). Diese Zusammenhänge nicht nur theoretisch, sondern auch methodisch-methodologisch fassbar zu machen, kann als eine wesentliche Herausforderung empirischer Forschung kenntlich gemacht werden, zumal das (post-)wohlfahrtsstaatliche Arrangement auch ,,von den sozialpädagogischen Akteuren permanent und kontinuierlich mit re-produziert, also hergestellt wird“" (Kessl und Otto 2008, S. 9 f.). 


\section{2 „Das Gesellschaftliche“ - eine poststrukturalistische Perspektivierung}

Seit einigen Jahren gewinnen poststrukturalistische Denkweisen und Versuche, diese Zusammenhänge auch empirisch fruchtbar zu machen, zunehmend an Bedeutung. Die Attraktivität entsprechender Zugänge gründet nicht zuletzt auf ihrem ,kritischen Potential“", welches eine radikale Reflexivität gegenüber unserer eigenen historischen Gewordenheit und die damit einhergehende Befangenheit einfordert. „Poststrukturalistische Ansätze“, so etwa Silke van Dyk (2012), ,,bieten eine theoretisch fundierte Delegitimierung der für die Geschichte des christlich-westlichen Abendlandes so zentralen Vorstellungen einer objektiv gegebenen, vom erkennenden Subjekt unterschiedenen, geschichtlich oder natürlich begründeten ,Wahrheit" oder ,Vernunft"“ (van Dyk 2012, S. 189). Diese kurze Auflistung wäre nun leicht um die vermeintliche Errungenschaft des Wohlfahrtsstaates zu ergänzen. Mit Blick hierauf scheint es uns notwendig, die Rede vom sozialstaatlichen Wandel gesellschaftstheoretisch einzuordnen und analytisch aufzufalten.

Aus poststrukturalistischer Perspektive ist es keineswegs zielführend, von der Gesellschaft als organizistischem Zusammenhang zu sprechen. Gegen eine entsprechende Uniformitäts- wie Universalitätsannahme wird das Gesellschaftliche vom Standpunkt der permanenten wie verstreuten (Re-)Formierung gedacht, gewissermaßen als eine unabschließbare (An-)Ordnung beweglicher Elemente, die in wechselseitig beeinflussender Beziehung und damit in Relationalität zueinander stehen. Es ist somit nicht das Moment der Statik, sondern ein unentwegtes Prozessieren im Spannungsfeld temporärer Stabilität und darauf bezogener Destabilisierung, welche ,das Gesellschaftliche“ kennzeichnet. Insofern hierbei, in poststrukturalistischer Logik, weder ein äußeres Strukturierungsmoment angenommen wird, noch gesellschaftliche Entwicklungen einem inneren, fundamentalen Eigenprinzip folgen, sind die (Re-)Produktionszusammenhänge des Gesellschaftlichen in diesem selbst liegend (vgl. van Dyk 2012), womit dann jeder Fortschritts- und Entwicklungsannahme zuerst einmal die Einsicht von Kontingenz entgegenzuhalten ist. Vor diesem Hintergrund sind auch sozialer Wandel und wohlfahrtsstaatliche Transformationsprozesse als Teil der für die Gesellschaft konstitutiven Instabilität zu verstehen (vgl. Gertenbach 2008). Unsere zu Beginn eingeführte Figur des ,wohlfahrtsstaatlichen Arrangements" wird nun sinnfällig, insofern sie eine temporäre KontingenzschlieBung (van Dyk 2012, S. 190) und damit die partielle Fixierung ,gesellschaftliche[r] Kräfteverhältnisse“ (Gertenbach 2008, S. 220) bedeutet. Wenn wir nun seit den 1970er Jahren die besagte „Krise der Wohlfahrtsstaatlichkeit“ diskutieren, ist diese als Problematisierung des wohlfahrtsstaatlichen Arrangements und der hierin aufzufindenden herrschaftsförmigen „Erstarrung und Blockierung der Machtverhältnisse“ (Moebius 2008, S. 165) zu lesen.

Entsprechend dieses poststrukturalistischen Vorbehalts gegenüber der Gesellschaft als eindeutig wie letztgültig bestimmbar, werden auch die hiervon abgeleiteten bzw. entsprechend kontextualisierten Denkweisen von Mikro-Makro ebenso wie von Struktur und Handlung, als allzu deterministische Konstruktionen, problematisch.

Als ertrag- und erkenntnisreich dürfte sich demgegenüber die Frage nach den gesellschaftlichen Machtverhältnissen erweisen. Der Blick auf die „Ökonomie der 
Machtbeziehungen“" verspricht verstehbar zu machen, in welchen Zusammenhängen und auf welche Weise sich das „Gesellschaftliche“ immer wieder (re-)formiert.

Einen möglichen poststrukturalistischen Zugriff auf die Analyse von Machtverhältnissen bietet sodann das Konzept der Gouvernementalität im Anschluss an Foucault, welches sowohl die individuenbezogene Menschenführung als auch die Regulierung von Bevölkerung zu denken gestattet. Die machtanalytische Erweiterung bestand für Foucault seinerzeit in der Relativierung der unmittelbaren Unterwerfung und einer stärkeren Betonung von Aspekten wie Freiheit bzw. der Eröffnung von Möglichkeitsräumen (Foucault 2005b, S. 287). Die Frage nach der „Kunst des Regierens“, welche den Kern der Foucaultschen Neuausrichtung seit den späten 1970er Jahren darstellte, fokussiert auf ein Interesse an den „Regierungspraktiken“, welche Methoden, Techniken und Programme der Leitung, Führung und Kontrolle von Individuen mit „Serien von Wissensarten“ (Foucault 2006a, S. 162) verknüpft. Was Foucault auf diese Weise zu explizieren versuchte, nämlich die Machtausübung auf der Grundlage von politökonomischem Wissen aufzuweisen, und das er schließlich auch auf die Dimension von „Selbstführungstechniken“ zu verlängern unternahm (vgl. Foucault 1989a, 1989b), fand in den sogenannten Gouvernementalitätsstudien einen fruchtbaren Niederschlag. Diese stellen mittlerweile ein umfangreiches Forschungsfeld dar, in dem zahlreiche Themen, wie pädagogische Ordnungen, Mitarbeiter*innenführung, bürgerschaftliche Engagements oder auch Strategien und Programme transnationaler Institutionen, wie der Europäischen Union, zum Gegenstand der Analyse gemacht werden (vgl. Bröckling und Krasmann 2010).

Insofern die Bevölkerung ,als Hauptzielscheibe“ der Machtausübung innerhalb von gouvernementalitätsanalytischen Zugängen gilt, ist es aber auch eine wesentliche Erkenntnis, dass ,die Menschen“ selbst als Co-Produzenten in diese Prozesse verstrickt sind und ihnen die Macht nicht als etwas rein Äußerliches begegnet. So ist es die zentrale These der an Foucaults Arbeiten anknüpfenden Studien zur Gouvernementalität, dass die gegenwärtigen Ordnungen des Sozialen und die mit ihr einhergehenden Regierungspraktiken weniger direkt auf Individuen abzielen und damit auf ihr Verhalten einwirken, sondern dass sich die Kunst der Regierung auf Bevölkerungsgruppen richtet und damit auf die Situationen und Kontexte, in denen Individuen handeln (Bröckling et al. 2000, S. 9).

\section{Diskurse und Praktiken - für eine empirische Vielfalt unter poststrukturalistischem Dach}

Der Poststrukturalismus lässt sich keineswegs als klar umrissene und einheitliche Theorie ausweisen. Allerdings kennzeichnet eine poststrukturalistische Haltung das Wissen um die Standortgebundenheit des eigenen Diskurses, der mitnichten unabhängig objektiv ist. Diskurse und Praktiken stellen das empirische Untersuchungsmaterial ,poststrukturalistischer Sozialwissenschaften“ und die „erste, kleinste oder letzte Einheit“" (Reckwitz 2008, S. 188) einer Analytik dar.

Klassische Bestimmungsversuche in der Vergangenheit wurden nicht müde, die Unvereinbarkeit bzw. Unvermittelbarkeit zwischen Diskursen und Praktiken zu betonen (Keller 2011; Reckwitz 2003), insofern nämlich Diskurse zumeist auf einer rein 
sprachlichen, Praktiken hingegen auf einer eindeutigen Handlungsebene lokalisiert wurden. In den letzten Jahren sind allerdings zahlreiche theoriesystematische Beiträge entstanden, die zwar auch noch Differenzen, vielleicht gar Oppositionen sehen, aber keineswegs mehr dem Nomos des unvereinbaren Gegensatzes folgen. Diskursund Praktikenforschung haben gewissermaßen, so Andreas Reckwitz (2008), damit begonnen, sich als ,zwei Seiten des gleichen [...] Analyseprojekts wahrzunehmen ohne einen - immer unbefriedigenden - ,Mittelweg' zu wählen“ (Reckwitz 2008, S. 190). Dabei scheint es unausgesprochener Konsens innerhalb dieser „Vermittlungsbemühungen“ zu sein, dass die jeweiligen theoretischen Fundierungsansprüche von Diskurstheorie und Praxeologie nicht aufgegeben werden, sondern eine systematische Relationierung von Gegenstandsbegründung, dem Umgang mit dem Material sowie der erkenntnispolitischen Anliegen angestrebt wird. Forschungskonzeptionen, in denen Diskurs- und Praktikenanalyse auf diese Weise reflexiv und mithin neu konturiert werden, liegen bereits vereinzelt vor: $\mathrm{Zu}$ nennen wären u. a. Daniel Wranas (2012a) Vorschlag zur Analyse „diskursiver Praktiken“, Andreas Reckwitz' (2008) Konzept der „Praktiken/Diskurs-Formationen“ oder auch Fabian Kessls (2011) Vorschlag zur Analyse von „Rationalisierungspraktiken“.

Die vier nun folgenden Skizzen methodologischer Zugänge im Kontext einer Diskurs-Praktiken-Forschung wählen verschiedene analytische Einsatzpunkte. Ein erster Zugriff bildet die (Neu-)Befragung des „Dispositivs“ als Analyserahmen für Diskurs/Praktiken-Formationen im Hinblick auf eine genealogische Geschichtsschreibung Sozialer Arbeit. Eine zweite Darstellung fokussiert sodann auf den analytischen Mehrwert einer Inblicknahme „diskursiver Praktiken“ im Kontext von „Programmanalysen“, bevor darauf folgend - in einer dritten Skizze - die vielmals vernachlässigte Dimension „,(mit)handelnder Artefakte“ in ethnographischen Untersuchungszusammenhängen thematisiert wird. Schließlich ist ein vierter Zugang daran interessiert, ein ethnographisches Forschungsinteresse in den Rahmen einer „Diskursivitätsanalytik“ zu stellen.

\subsection{Das Dispositiv im Zusammenhang einer Diskurs-Praktiken Forschung}

Ausgehend von einer genealogischen Geschichtsschreibung Sozialer Arbeit wird die Frage nach den gegenwärtigen Transformationsprozessen Sozialer Arbeit im Sinne einer "Geschichte der Gegenwart" historisiert, um hierdurch die Gegenwart in ihrer Gewordenheit untersuchen zu können. Das Verfahren der genealogischen Geschichtsschreibung ist zum einen anschlussfähig an die Positionen einer machtanalytischen Diskurs-Praktiken-Forschung, schließt aber zugleich an poststrukturalistische Versuche an, Gesellschaft nicht als Einheit zu denken, sondern im Sinne einer ,aufsteigenden Analyse“ von den „lokalen Erinnerungen“ auszugehen, um die Konstellationen in den Blick zu nehmen, aus denen sich diese Ordnungen zusammensetzen (vgl Moebius und Reckwitz 2008; Bröckling et al. 2004).

Mit dem Verzicht auf eine Zentralperspektive auf Gesellschaft verschiebt sich auch der Blick der historischen Untersuchung: „Wenn bestimmte Objekte sich nicht mehr ,von selbst verstehen“, dann ist es erforderlich, das Netz von Verbindungen, Kräfteverhältnissen, Blockaden, Strategien, Zirkularität etc. ausfindig zu machen, das es erlaubt, zu einem gegebenen Zeitpunkt das zu etablieren, was als selbstver- 
ständlich, universell und notwendig gilt [- so hätte beispielsweise die Soziale Arbeit; Einf. d. Verf.*innen] nicht mehr den Status eines Ausgangspunktes der Analyse, sondern [erscheint] als Effekt von historischen Praktiken“" (Lemke 2011, S. 334).

Die Ablehnung einer den Dingen vorgängigen Struktur und die Annahme von Kontingenz eröffnet den Raum für die Frage, wie denn auf diese Weise Gesellschaft historisch zu denken ist. Die Figur des „Dispositivs“, welches als relationale (An-)Ordnungseinheit von konstitutiven gesellschaftlichen Elementen verstanden werden kann, dient dabei als Brückenschlag zwischen Geschichtsschreibung (Kontingenzannahme) und Machtthematik. Michel Foucault bezeichnet als „Dispositiv“ ein Netz von heterogenen Elementen. Dieses heterogene Ensemble, wie es in einem Interview aus der Mitte der 1970er Jahren heißt, könne aus Diskursen, Institutionen, architektonischen Einrichtungen, reglementierenden Entscheidungen, Gesetzen, administrativen Maßnahmen und wissenschaftlichen Aussagen bestehen (Foucault 2003c, S. 392 ff.). Das Dispositiv sei folglich ein Netz zwischen Materialitäten und Programmen, „Gesagtem wie Ungesagtem“. Dabei geht es aus der Perspektive einer Geschichtsschreibung insbesondere um die Verbindungen zwischen diesen Elementen und deren Wandel. Das Dispositiv stellt „Verwirklichungsbedingungen von Macht dar, also die Bedingungen, unter denen sich spezifische Machteffekte realisieren können. Zugleich werden diese Strukturen nur in der gesellschaftlichen Praxis realisiert, wobei die Struktur eines Dispositivs niemals sämtliche Effekte determinieren kann, [...] - so ist es möglich, dass Wirkungen entstehen, die über das Dispositiv hinausweisen“ (Meissner 2010, S. 141).

In dieser Form können Ordnungszusammenhänge gesellschaftlicher Phänomene sowie deren strukturelle ,Verfestigung“ und „Gleichgerichtetheit“ untersucht werden (Meissner 2010, S. 138), ohne eine transzendentale Begründung oder eine ,überhistorische Geschlossenheit der Struktur" behaupten zu müssen (Meissner 2010, S. 139). Das Dispositiv ermöglicht eine Geschichtsschreibung, welche in der Lage ist, „historischen Wandel jenseits von teleologischen oder dialektischen Theorien in seiner gesamten temporalen Heterogenität zu konzeptionalisieren“ (Schauz 2010, S. 106) und damit Diskontinuitäten wie Brüche in den Mittelpunkt der Analyse zu rücken. Verschiedene Zeitschichten von unterschiedlicher Dauer und mit unterschiedlicher Geschwindigkeit verweisen auf die Ungleichzeitigkeit der Geschichte. Damit werden Verfestigungen und Trägheiten von Materialität und Institutionen zu historisch spezifischen Zeitpunkten - neben sich beispielsweise permanent aktualisierenden Praktiken und Diskursen - zugleich als Elemente eines Dispositivs denkbar (Schauz 2010, S. 107).

Das Konzept des Dispositivs vermittelt somit zwischen diskurstheoretischen als auch praxeologischen Perspektiven, dem „Gesagten“ wie dem „Ungesagten“, dem „Sagbaren“ und dem „Sichtbaren“ (Foucault 2003c, S. 392) und ermöglicht, sie in eine gemeinsame Analytik zu integrieren. Dabei sind Praktiken und Diskurse aufeinander verwiesen, ,[d]enn es gibt in der Tat keine ,Praktiken“ ohne ein bestimmtes Regime der Rationalität“ (Foucault 2005a, S. 33). Sie sind trotzdem nicht dasselbe, das Eine ist auch nicht auf das Andere zurückzuführen; sie ,durchdringen sich wechselseitig [...]. Beide Formen setzen sich wechselseitig voraus“ (Deleuze 1987), das Dispositiv ist ein „Gebilde des Zusammenschlusses“. 


\subsection{Programme als diskursive Praktiken}

Die nun folgende Skizze befasst sich mit der Frage, wie Programme auch als Praktiken betrachtet werden können und welche methodologischen Konsequenzen sich hieraus ergeben, also wie eine Relationierung von Diskursen und Praktiken für eine Analyse politischer Programmpapiere heuristisch eingefasst werden kann. Programme, so ließe sich formulieren, vermitteln zwischen einer politischen Denkweise und deren Implementierung bzw. Umsetzung in eine Praxis. In gouvernementalitätstheoretischer Perspektive, wie sie Foucault (2006a, 2006b) in seinen Vorlesungen vorgeschlagen hat, lassen sich Programme als Effekte von gesellschaftlichen Kräfteverhältnissen verstehen. Programme nehmen ihren Ausgangspunkt in Prozessen der Problematisierung, sie konstruieren Probleme, die auf eine bestimmte Weise gerahmt und mit Lösungsvorschlägen versehen werden (vgl. Bröckling et al. 2004). Diese „Formen der Problematisierung“ sowie die damit verbundenen ,Strategien der Bearbeitung" bilden einen zentralen Ansatzpunkt von Programmanalysen.

Eine Kritik an gouvernementalitätsanalytischen Studien betont die Möglichkeit eines darin enthaltenen programmanalytischen Reduktionismus (vgl. Kessl 2007), der die sozialen Praktiken auf ein Gegenüber reduziere und Gefahr liefe, vorschnell auf eine deterministische Wirkung von Programmen zu schließen. Auch wenn die verschiedenen Kritiken an Gouvernementalitätsstudien nicht einfach von der Hand zu weisen sind, begründen sich einige Einwände doch in der Annahme einer vorhandenen Dualität von Makro/Mikro respektive Struktur/Handeln. Gouvernementalitätsanalysen werden dann als „bloß programmatische, also diskursive Analyse [bezeichnet, die] auf einer programmatischen, textanalytischen Ebene verbleiben“ (Bührmann und Schneider 2007). Diese hier zugrundeliegende Vorstellung von einfach gesprochen: Programm ist Text ist nicht Praktik ist so nicht aufrechtzuerhalten. Gleichzeitig ist ebenfalls nicht von einer einfachen Wirkung von Programmen auszugehen, die - vielleicht nicht ungebrochen, doch wirksam - in Praktiken eingingen, so dass diese bloß einen Spiegel programmatischer Strategien darstellen.

In gouvernementalitätstheoretischen Zugängen (vgl. Kessl und Krasmann 2005) wird daher für eine Perspektivierung plädiert, die Programme nicht als Makroinstanzen entwirft, sondern als (Regierungs-)Praktiken in den Blick nimmt, die Einfluss haben auf die Möglichkeiten, Wirklichkeit begreiflich zu machen und zu konstituieren (Wahrheitsspiele). Diese Perspektivierung versteht Programme selbst als Praktik, oder genauer, möchte sie als machtförmige diskursive Praktiken innerhalb von Kräfteverhältnissen in den Blick nehmen, wie es Gouvernementalitätsanalysen diskursiver Praktiken vor dem Hintergrund von praxeologisch-poststrukturalistischen Überlegungen bereits angelegt haben (u. a. Scharl und Wrana 2014; Langer und Wrana 2010; Ott und Wrana 2010). Diese gehen davon aus, dass Wirklichkeit im Modus praktischer Vollzüge hergestellt wird und konzipieren Diskursivität ausgehend vom Vollzug diskursiver Praktiken (Ott und Wrana 2010, S. 155f; Scharl und Wrana 2014, S. 350).

Fasst man Programmtexte als diskursive Praktik auf, dann geraten in der Analyse die Vollzugsweisen derartiger Sprechakte sowie die spezifische Artikulationsform von Programmen in den Blick. Programme produzieren demnach durch Sprache Wahrheitseffekte, formen weitere Praktiken und lassen bestimmte Objekte denk- 
und sagbar werden. Die Differenz von anvisierten Programmen und so genannten „realen Effekten“ lässt sich damit nicht aufrechterhalten. Programme stellen in dieser Lesart Probleme (und Lösungen) als diskursive Gegenstände her (vgl. Ott und Wrana 2010). Regierungspraktiken sind demnach mehr als „Produktionsstätten“ von Programmen. Regieren kann dann als Gesamtheit von Sprechakten verstanden werden, welche in ihrem Vollzug Politikfelder, politische Handlungsfelder, Subjektpositionen, Steuerungsoptionen (ver-)schließen, initiieren, ignorieren, (de-)legitimieren usw., also überhaupt erst als solche hervorbringen.

Regieren als Prozess der Artikulation zu fassen, bildet dann den Untersuchungsgegenstand in der Analyse von politischen Programmpapieren. Die diskursiven Praktiken der politischen Artikulationen erscheinen so als heterogen, fragil und in diskursiven Kämpfen produziert. Der Blick richtet sich dann auf den diskursiven Kampf in den politischen Artikulationen sowie die Stabilisierung und Destabilisierung von Diskurspositionen. Diese Perspektivierung ermöglicht es, im Kontext programmanalytischer Transformationsforschung Sozialer Arbeit auf der Ebene von Governance die Beschränkung auf die Beschreibung von Steuerungs- und Verwaltungswissen zu überwinden. Eine solche Situierung von Programmen als diskursive Praktiken erlaubt weiterhin eine herrschaftskritische Gesellschaftsanalyse und Aussagen zu konkreten politischen Kontexten wie Strategien - so fluide und unbestimmt diese auch zu sein scheinen.

\subsection{Artefakte als Partizipanden in Praktiken}

Neben einer angemessenen Relationierung von Praktiken und Diskursen ergeben sich auch Fragen hinsichtlich der Relationierung menschlicher Akteur*innen und der dinglichen Welt in den Praktiken. In einer solchermaßen erweiterten Perspektive ginge es darum, ,die variablen Bedingungen des Vollzugs der Praxis situationsanalytisch zu identifizieren, also das Zusammenkommen und -wirken von sozialisierten Körpern mit materiellen Artefakten und Dingen sowie mit diskursiven und symbolischen Formationen zu untersuchen“ (Hillebrandt 2015, S. 17).

Für die Analyse des Zusammenwirkens von Menschen und Dingen liegen verschiedene sozialtheoretische Theorieangebote vor: etwa die Akteur-Netzwerk-Theorie Bruno Latours (2007), die von einer Symmetrie und prinzipiellen Gleichwertigkeit menschlicher und nicht-menschlicher Aktanten in den Akteur-Netzwerken ausgeht, oder die Konzeption einer ,distributed action“ (Rammert 2007, S. 88) und graduierten Handlungsträgerschaft von Technik bei Werner Rammert und Ingo Schulz-Schaeffer (2002). Forschungsmethodologisch und -methodisch stellt sich hier die Frage und Aufgabe, wie das Mithandeln und -wirken der Dinge angemessen $\mathrm{zu}$ erfassen ist, also wie „Menschen, Organismen, Dinge und Artefakte wie Werkzeuge, technische Apparaturen, Sprache und Bilder [...] sich hier in sozialen Praktiken so miteinander [verwickeln], dass sie einen Kontext füreinander bilden, in dem sie intelligibel werden, und zugleich eine unterscheidbare soziale Ordnung etabliert wird“" (Alkemeyer 2013, S. 43).

Klassische Perspektiven, die den Fokus der Forschung auf die Nutzung von Werkzeugen, Technik, Medien etc. durch Menschen (Fachkräfte, Adressat*innen etc.) legen, sollten dabei insofern eine Erweiterung erfahren, als es nicht nur um „Praktiken 
mit Dingen“, sondern auch um die „Praktiken der Dinge“ (Bollig 2008) geht. Artefakte werden in dieser Perspektive also als Partizipanden in Praktiken betrachtet. Mit Blick auf die Interaktion von materiellen und technischen Artefakten und Menschen werden damit weder technikdeterministisch noch strukturalistisch-zuschreibend die Menschen als diejenigen konstruiert, die sich der Technik bedienen bzw. die Technik als determinierende Rahmung für Arbeitsvollzüge und Kommunikationen bestimmt. $\mathrm{Zu}$ untersuchen wäre in Kontexten Sozialer Arbeit, wie innerhalb geteilter Praktiken von menschlichen Akteuren und technisch-medialen Artefakten etwa Problemdefinitionen und Fallkonstitutionen (Wahrheitseffekte) stattfinden, wie sich innerhalb der Settings, unter Einbezug von Artefakten, die Akteur*innen zueinander positionieren und interagieren und welche Praxisformen dabei sichtbar werden. Dabei wären etwa berufliche - Praktiken in einer Verbindung von Feldforschung und objektorientierter Analyse zu untersuchen, die sowohl die Praktiken mit, als auch die Gestalt und Konstruktion sowie den Gebrauch von digitalen wie analogen Artefakten in den Blick nimmt. Für die forschungsmethodische Praxis der teilnehmenden Beobachtung lässt sich dabei das Postulat ethnographischer Feldforschung des ,following the actor" beispielsweise für die digitalisierten Formen beruflicher Praktiken als ,,shadowing software“ übersetzen (Bruni 2005). Wie den menschlichen Akteur*innen folgt man dabei den Wegen, die die Daten und Informationen bspw. innerhalb einer Fach-Software nehmen: wie sie von der ihre Fachleistungsstunde dokumentierenden Fachkraft in die zur Abrechnung in die Verwaltung ,wandern“ oder wie sie in der Anwendung verarbeitet, zusammengeführt und schließlich verdichtet als Controllinginformation der Leitungskraft zur Verfügung gestellt werden.

In einer solchen Ethnographie von Praktiken der Akteur*innen und Artefakte im ,doing Sozialer Arbeit“ würde eine praxistheoretische Perspektive auf Handeln und auf das Soziale zugrunde gelegt, wie sie von Reckwitz (2003) als verbindende Synthese unterschiedlicher Theorie- und Forschungsprogramme praxeologischer Orientierung herausgearbeitet wurde, ,um die Routinen in Unternehmen, die Formen der Verwendung technischer und medialer Artefakte, die Charakteristika geschlechtlicher ,performances“ oder etwa das „,doing culture“ in alltäglichen Zeitpraktiken zu rekonstruieren“ (Reckwitz 2003, S. 282). Die von ihm skizzierte „Praxeologie“ kann als ein „Forschungsprogramm für die materiale Analyse“ (Reckwitz 2003, S. 284) dienen, in der Objekte eine herausgehobene Stellung einnehmen. Das Soziale konstituiert sich in einem solchen Verständnis über Praktiken, verstanden als „Komplex aus regelmäßigen Verhaltensakten und praktischem Verstehen [die sich] durch die materiellen Instanzen“ (Reckwitz 2003, S. 290) menschlicher Körper und nicht-menschlicher Artefakte realisieren.

Es geht dabei weder um ein ,,präpraktisches Subjekt‘, das dem Handlungsvollzug logisch und genealogisch als unabhängige Ursache vorausgeht, noch [um] eine von Strukturen, Praktiken oder Diskursen abzuleitende Größe, sondern [das handelnde Subjekt] konstituiert, organisiert und zeigt sich in menschlicher Koexistenz im Rahmen sogenannter ,Sites of the Social'. Dabei handelt es sich um Stätten, die menschliches und nichtmenschliches Handeln auf spezifische Weise kontextualisieren [...]. Das Site-Konzept bringt damit sowohl die ,volle Bandbreite' möglicher Beziehungen zwischen ontisch verschiedenen Partizipanden als auch die Gleichzeitigkeit von Stabilität (Regelmäßigkeit) und Instabilität (Unregelmäßigkeit) in den 
Blick“ (Alkemeyer 2013, S. 43). Handlungen sind aus dieser Perspektive ,als aus ,dem Spiel' heraus sich ergebende Interventionen, die weitere Handlungen anstoßen und Begebenheiten geschehen machen“, zu verstehen (Alkemeyer 2013, S. 47). Praktiken können damit als ,ein dynamisches Gefüge von Beziehungen zwischen (Subjekt-)Positionen und Handlungen betrachtet“ und in ihrer Multiperspektivität analysiert werden (Alkemeyer 2013, S. 48). Diese praxistheoretische Perspektive eröffnet die Möglichkeit, interagierende Menschen, Technik, institutionelle Strukturen und größere Rahmungen offen in den Blick zu nehmen und die sich dabei entfaltenden Praktiken, Brüche, Widersprüche und Positionierungen der Akteur*innen beobachtbar und analytisch fassbar zu machen.

\subsection{Ethnographische Diskurs(ivitäts)analytik}

Die ,ethnographische Diskursanalyse“ kann als ein weiterer, mittlerweile eigenständiger Zugang innerhalb des Feldes poststrukturalistischer Diskursforschung angesehen werden (Macghilchrist et al. 2014; Ott et al. 2014, 2012). Die Attraktivität des Forschungsansatzes lässt sich nicht nur, aber auch in der systematischen Weiterentwicklung ethnographischer Forschungsdesigns ausmachen, als dass diese nun konsistenter gesellschaftstheoretisch rückzubinden sind. Innerhalb des hier diskutierten Zusammenhangs ,,(post-)wohlfahrtsstaatlicher Transformationsforschung“ erlauben diskursethnographische Zugänge u. a. die konsequente Inblicknahme ,sozialpädagogischer Erbringungssettings“, um sie auf die Effekte „,neosozialer Programmierung“ hin zu untersuchen, ohne dabei eine Struktur-Handlungs-Dichotomie vorauszusetzen. An die Stelle eines unmittelbaren, intendierten Wirkungszusammenhangs wohlfahrtsstaatlicher Programmatik auf die Praxis Sozialer Arbeit rückt die Überzeugung relationaler Beziehungen und die Forderung, diese in einem reflexiven Analyserahmen einzufangen.

Eine ethnographische Diskursanalyse nimmt (für gewöhnlich) ihren Ausgangspunkt bei der Frage nach den situierten Praktiken innerhalb des zu untersuchenden Feldes. Vor dem Hintergrund einer poststrukturalistisch begründeten Skepsis gegenüber dem Subjekt als Sinnstiftungszentrum werden Praktiken hier nicht von den Akteur*innen her gedacht und auch nicht an die Körper als Erzeugungsprinzip geknüpft (vgl. Hirschauer 2004), sondern als diskursive und damit performative Praktiken konturiert. In Übereinstimmung mit poststrukturalistischen Grundannahmen wird die Sinnproduktion dabei als Funktion betrachtet, insofern Materialität, Wissensobjekte, Bedeutungsfelder und Subjektivitäten (vgl. Wrana 2006; Foucault 1981) in Bezug zueinander gesetzt werden. Die Frage nach der Materialität, als die Medialität diskursiver Praktiken, wird dabei innovativ geweitet. Es sind nicht nur und ausschließlich gegebene (Archiv-)Texte, die das Untersuchungsmaterial darstellen, sondern dieses kann im Rahmen des Forschungsprozesses auch selbst hervorgebracht werden. Zugrunde gelegt wird hierbei die Einsicht, dass das „Diskursive“ nicht nur Sprachliches umfasst, sondern dessen Verständnis auf ,,alle Signifikationssysteme“ (Laclau 2007, S. 30) zu erweitern ist. Der „Analysekorpus“ kann so, neben (programmatischen) Texten und institutionellen Papieren, auch ethnographische Beobachtungsprotokolle, Interviewtranskripte, Abschriften von Gruppendiskussionen und selbst Bilder umfassen, mithin also ,alles, was in der Lage ist, zu einem Zei- 
chen zu werden“ (Langer 2008, S. 55). In den Blick von Diskursanalyse geraten so auch ethnographisch beschreibbares Körperhandeln, Raum(an)ordnungen sowie der Umgang mit Artefakten.

Streng genommen ist die „ethnographische Diskursanalyse“ keine genuine Diskursanalyse, denn zum einen werden keine (einzelnen) Diskurse untersucht, sondern Diskursivität, zum anderen wird ein ethnomethodologischer Bezugsrahmen aufgespannt, der entsprechende Untersuchungen deutlich auch als Praktikenforschung (an)erkennen lässt. Die Verschiebung des Interesses auf die Frage der Diskursivität geht mit der Einsicht einher, dass Diskurse als Ensemble diskursiver Praktiken zu verstehen sind, die nur in historischer Perspektive als Formationen rekonstruiert werden können. Fokussiert man aber - aus ethnographischer Perspektive - auf beobachtbare Vollzugspraktiken, so wird man mit der Vielschichtigkeit und Unabgeschlossenheit von (diskursiven) Wissensordnungen konfrontiert (vgl. Wrana 2012b).

In einer Art Novellierung der ethnomethodologischen Perspektive des „doing“ (vgl. West und Zimmerman 1987) wird davon ausgegangen, dass institutionelle „Rahmungen“ immer praktisch gefüllt werden müssen, was diskurstheoretisch übersetzt auf die Kennzeichnung ,programmatischer Vorgaben“ als stets überdeterminierte Anrufungsszenarien hinausläuft (vgl. Kessl und Krasmann 2005). Situatives Handeln lässt sich in diesem Sinne auch als ein „doing discourse“ (vgl. Füssel und Neu 2010) verstehen, das nicht den Vorgaben einer Programmatik folgt, sondern eingedenk unterschiedlich verfügbarer Wissensordnungen - die vermeintlich strukturelle Ordnung immer wieder verschiebt, uminterpretiert oder unterläuft. Zugrunde gelegt ist diesem Verstehenszusammenhang die Logik des Iterationstheorems im Anschluss an Jacques Derrida (2001) und Judith Butler (2006), das davon ausgeht, dass Praktiken kein Ursprungsdenken auszeichnet, sondern jede Praktik als kontextuell aktualisierende Zitation anderer Praxen zu verstehen ist.

Gegenüber einem dualistischen Mikro/Makro-Verständnis wird auf diese Weise die spannungsreiche Frage nach den Machtverhältnissen in Hinblick auf die verhandelten bzw. verhandelbaren Wissensordnungen und die damit einhergehenden dynamischen Machtbeziehungen aufgeworfen. Programmatische bzw. institutionelle Rahmungen werden so zum einen als Ermöglichungs- bzw. Verunmöglichungskontexte dechiffrierbar, zum anderen lassen sie sich als „Arenen“ diskursiver Kämpfe beobachten. Vollzugspraxen und strukturelle Ordnungen stehen sich hierbei weder unvermittelbar gegenüber, noch kennzeichnet sie ein deterministisches Verhältnis. Vielmehr handelt es sich um Praktiken unterschiedlicher Materialität, die in relationalen Bezugsverhältnissen zueinanderstehen und detailliert rekonstruiert werden müssen. Diskursive Ethnographien können so unterschiedlichen Erkenntnisinteressen nachgehen. Fragen nach den (un)möglichen Subjektivierungsweisen sind ebenso statthaft wie die nach der Hervorbringung eines verhandelten diskursiv Gegenständlichem oder nach den Konfliktlinien, die einem zu untersuchenden sozialpädagogischen Handlungsfeld immanent sind. Kennzeichnend für die Forschungspraxis ist gewissermaßen die Inblicknahme eines überschaubaren Kontextes, um hier die lokalen, situativen (Aus-)Handlungsweisen als facettenreiche Geltendmachung von Macht-Wissen-Zusammenhängen zu rekonstruieren. 


\subsection{Praxeologische und diskursive Forschung zur Transformation des Wohlfahrtsstaats in machtanalytischer Perspektive}

In der jüngeren Vergangenheit haben praxeologische und diskursanalytische Lesarten in der Sozialen Arbeit eine sichtbare Aufwertung erfahren. Diese Entwicklung resultiert zum einen aus der innerhalb der Disziplin erkennbar stärkeren Hinwendung zu empirischen Forschungsarbeiten, zum anderen - und damit durchaus verknüpft einer kritischen Befragung des disziplinären Selbstverständnisses. Vor dem Hintergrund poststrukturalistischer und machtanalytischer Zugänge haben wir im Beitrag den Versuch unternommen, den möglichen analytischen Mehrwert praxeologischer und diskursanalytischer Forschungsstrategien im Kontext einer „Transformationsforschung Sozialer Arbeit" herauszuarbeiten. Die hier skizzierten methodologischen Zugänge erlauben die Analyse und Rekonstruktion von Prozessen, in denen Soziale Arbeit als Akteurin innerhalb des (post-)wohlfahrtsstaatlichen Arrangements agiert und an dessen Herstellung und Umformung mitwirkt.

Operiert eine Diskurs/Praktiken-Forschung auf Grundlage eines poststrukturalistisch informierten Gesellschaftsverständnisses und geht damit von einer konstitutiven Unabschließbarkeit und der Relationalität des Gesellschaftlichen aus, entsteht die Herausforderung, dass die Gesellschaft einerseits als kontingente Bezugsgröße als unmöglich gedacht werden kann, das Gesellschaftliche aber andererseits als Reflexionszusammenhang notwendig ist, ,wenn das Soziale nicht in gleich-gültige Partikularitäten zerfallen soll“" (van Dyk 2012, S. 187). Als Bezugspunkte eines relationalen Gesellschaftsverständnisses rücken dann v. a. die temporären Stabilisierungen, partiellen Fixierungen und historisch-spezifischen Verfestigungen sozialer Verhältnisse und gesellschaftlicher (An-)Ordnungen in den Blick, die als Effekte gesellschaftlicher Kräfteverhältnisse zu verstehen sind. Wir haben im Rahmen unserer Darstellung versucht, das ,,postwohlfahrtsstaatliche Arrangement“ als Rahmen aufzuspannen, der ein Transformationsgeschehen anerkennt, dieses aber nicht auf der staatlichen Ebene verankert, sondern als Problematisierung von Wohlfahrtsstaatlichkeit fasst. Wohlfahrtsstaatlichkeit wurde dabei als eine spezifische, kontingente Macht-Wissen-Ordnung und Regierungsweise ausgedeutet, die gegenwärtig ganz unterschiedlich bearbeitet wird. Das ,wie“ dieser Bearbeitung lässt sich gleichwohl nicht aus einem übergeordneten wie auch nicht aus einem immanenten Strukturierungsprinzip ableiten, sondern muss jeweils kontextbezogen in den Blick genommen werden. Damit wird auch die analytische Frage nach Macht- und Herrschaftsverhältnissen aufgerufen, wobei diese einer jeweils gegenstands- und erkenntnisinteressebezogenen Konkretisierung bedarf, um sie ,nicht [nur] als abstrakt-subversives Potenzial zu kultivieren“ (van Dyk 2012, S. 201) und dadurch gleichsam ihr kritisches Potenzial zu gefährden. Der alleinige Verweis auf Macht- und Herrschaftsverhältnisse ist darum analytisch wenig aufschlussreich und muss jeweils dahingehend ausbuchstabiert werden, auf welche Weise sich in den jeweiligen Zugängen einer Diskurs-Praktiken-Forschung Macht- und Herrschaftsverhältnisse untersuchen und sichtbar machen lassen.

Die hier skizzierten methodologischen Optionen greifen vor diesem Hintergrund in unterschiedlicher Weise auf die machtanalytische Perspektive der „Gouvernementalität“" zurück und fokussieren auf differente Weise die Praxis der Machtausübung 
(Regierungspraktiken) im Kontext Sozialer Arbeit. Damit werden u. a. mikroskopische Inblicknahmen von Zusammenhängen möglich, in denen die Machtausübung als ein ,auf Handeln gerichtetes Handeln“ und damit die „Regierung“ von Menschen durch andere Menschen beschrieben werden kann (Foucault 2005b, S. 287).

Über den Begriff der Regierung, wie er oben skizziert wurde, lassen sich so (neosoziale) Staatsformierung und Subjektivierung in einer gemeinsamen Perspektive untersuchen. Das Erkenntnisinteresse richtet sich dann auf die Taktiken und Mit$t e l$, mit denen dieser Zweck erreicht wird bzw. erreicht werden soll. Hierüber wäre beispielsweise kenntlich zu machen, wie Herrschaftstechniken sich verschiedener Verfahren und Prozesse bedienen, so dass Individuen oder Gruppen der Bevölkerung auf sich selbst einwirken, sich selbst regulieren (Bröckling et al. 2000, S. 29). Der Verweis auf die Mittel lenkt den Blick auf die technologischen Aspekte, also wie sich beispielsweise Praktiken der Regierung mit Hilfe von Verfahrensweisen in Arrangements oder Artefakten materialisieren, dabei mit spezifischen Wissensbeständen verknüpfen und hierüber konkrete Künste der Fremd- und Selbstführung institutionalisiert werden.

Anders konstelliert, gleichwohl im gleichen Fahrwasser bewegt sich ein Interesse an der Frage von (Wohlfahrts-)Staatsformierung, bei dem nicht der Staat selbst den Ausgangspunkt darstellt. Unsere hier entfalteten Perspektivierungen auf die Gouvernementalität reflektieren allesamt auf die Frage nach der ,(Neu-)Formierung des Sozialen“, die wir mit Blick auf Soziale Arbeit und ihre Verstrickung unter der Perspektive eines Wandels des „,wohlfahrtsstaatlichen Arrangements“ verhandeln, sodann aber vor dem Hintergrund je eigener Untersuchungsfelder und Erkenntnisinteressen unterschiedlich ausbuchstabieren. Eine so verstandene machtanalytische Erweiterung von Forschungsprogrammen konstituiert nicht nur einen spezifischen Untersuchungsgegenstand, gleichsam lässt sich hierüber auch ein spezifisches Erkenntnisinteresse anzeigen.

Danksagung Wir möchten uns an dieser Stelle ausdrücklich bei Christian Gräfe (Universität Duisburg-Essen) sowie den anonymen Gutachter*innen der Sozialen Passagen für ihre wertvollen kritischen Hinweise bedanken.

Open Access Dieser Artikel wird unter der Creative Commons Namensnennung 4.0 International Lizenz (http://creativecommons.org/licenses/by/4.0/deed.de) veröffentlicht, welche die Nutzung, Vervielfältigung, Bearbeitung, Verbreitung und Wiedergabe in jeglichem Medium und Format erlaubt, sofern Sie den/die ursprünglichen Autor(en) und die Quelle ordnungsgemäß nennen, einen Link zur Creative Commons Lizenz beifügen und angeben, ob Änderungen vorgenommen wurden.

\section{Literatur}

Alkemeyer, T. (2013). Subjektivierung in sozialen Praktiken. Umrisse einer praxeologischen Analytik. In T. Alkemeyer, G. Budde \& D. Freist (Hrsg.), Selbst-Bildungen. Soziale und kulturelle Praktiken der Subjektivierung (S. 33-68). Bielefeld: transcript.

Bollig, S. (2008). „Praktiken der Instrumentierung“. Methodologische und methodische Überlegungen zur ethnografischen Analyse materialer Dokumentationspraktiken in kinderärztlichen Vorsorgeuntersuchungen. Zeitschrift für Sozialisationsforschung und Erziehungssoziologie, 28, 301-315.

Bröckling, U. (2007). Das unternehmerische Selbst. Soziologie einer Subjektivierungsform. Frankfurt am Main: Suhrkamp.

Bröckling, U., \& Krasmann, S. (2010). Ni méthode, ni approche. Zur Forschungsperspektive der Gouvernementalitätsstudien - mit einem Seitenblick auf Konvergenzen und Divergenzen zur Diskurs- 
forschung. In J. Angermüller \& S. van Dyk (Hrsg.), Diskursanalyse meets Gouvernementalitätsforschung. Perspektiven auf das Verhältnis von Subjekt, Sprache, Macht und Wissen (S. 23-42). Frankfurt am Main: Campus.

Bröckling, U., Krasmann, S., \& Lemke, T. (2000). Gouvernementalität der Gegenwart. Studien zur Ökonomisierung des Sozialen. Frankfurt am Main: Suhrkamp.

Bröckling, U., Krasmann, S., \& Lemke, T. (2004). Einleitung. In U. Bröckling, S. Krasmann \& T. Lemke (Hrsg.), Glossar der Gegenwart (S. 9-16). Frankfurt am Main: Suhrkamp.

Bruni, A. (2005). Shadowing software and clinical records: on the ethnography of non-humans and heterogeneous contexts. Organization, 12, 357-378.

Bührmann, A. D., \& Schneider, W. (2007). Mehr als nur diskursive Praxis? - Konzeptionelle Grundlagen und methodische Aspekte der Dispositivanalyse. Forum Qualitative Sozialforschung, 8, 28.

Butler, J. (2006). Hass Spricht. Zur Politik des Performativen. Frankfurt am Main: Suhrkamp.

Dahme, H.-J., \& Wohlfahrt, N. (2005). Aktivierende Soziale Arbeit. Theorie - Handlungsfelder - Praxis. Baltmannsweiler: Schneider.

Dahme, H.-J., \& Wohlfahrt, N. (2008). Der Effizienzstaat: die Neuausrichtung des Sozialstaats durch Aktivierungs- und soziale Investitionspolitik. In B. Bütow, K. A. Chassé \& R. Hirt (Hrsg.), Soziale Arbeit nach dem sozialpädagogischen Jahrhundert. Positionsbestimmungen Sozialer Arbeit im Post-Wohlfahrtsstaat (S. 43-59). Opladen: Budrich.

Dahme, H.-J., Otto, H.-U., Trube, A., \& Wohlfahrt, N. (2003a). Soziale Arbeit für den aktivierenden Staat. Opladen: Leske + Budrich.

Dahme, H.-J., Otto, H.-U., Trube, A., \& Wohlfahrt, N. (2003b). Einleitung. In H.-J. Dahme, H.-U. Otto, A. Trube \& N. Wohlfahrt (Hrsg.), Soziale Arbeit für den aktivierenden Staat (S. 9-13). Opladen: Leske + Budrich.

Dahme, H.-J., Trube, A., \& Wohlfahrt, N. (2008). Soziale Arbeit für den aktivierenden Staat. In Bielefelder Arbeitsgruppe 8 (Hrsg.), Soziale Arbeit in Gesellschaft (S. 268-275). Wiesbaden: VS.

Deleuze, G. (1987). Foucault. Frankfurt am Main: Suhrkamp.

Derrida, J. (2001). Limited Inc. Wien: Passagen.

Dollinger, B., \& Raithel, J. (2006). Aktivierende Sozialpädagogik. Ein kritisches Glossar. Wiesbaden: VS.

Donzelot, J. (1994). Die Förderung des Sozialen. In R. Schwarz (Hrsg.), Zur Genealogie der Regulation. Anschlüsse an Michel Foucault (S. 109-160). Mainz: Decaton.

van Dyk, S. (2012). Poststrukturalismus. Gesellschaft. Kritik. Über Potenziale, Probleme und Perspektiven. Prokla, 167(42), 185-210.

Foucault, M. (1981). Archäologie des Wissens. Frankfurt am Main: Suhrkamp.

Foucault, M. (1989a). Der Gebrauch der Lüste. Sexualität und Wahrheit. Bd. 2. Frankfurt am Main: Suhrkamp.

Foucault, M. (1989b). Die Sorge um sich. Sexualität und Wahrheit. Bd. 2. Frankfurt am Main: Suhrkamp.

Foucault, M. (2003a). Erläuterungen zur Macht. Antworten auf einige Kritiker. In M. Foucault (Hrsg.), Schriften (Bd. III, S. 784-795). Frankfurt am Main: Suhrkamp.

Foucault, M. (2003b). Die analytische Philosophie der Politik. In M. Foucault (Hrsg.), Schriften (Bd. III, S. 675-695). Frankfurt am Main: Suhrkamp.

Foucault, M. (2003c). Das Spiel des Michel Foucault. In M. Foucault (Hrsg.), Schriften (Bd. III, S. 391-432). Frankfurt am Main: Suhrkamp.

Foucault, M. (2005a). Diskussion vom 20. Mai 1978. In M. Foucault (Hrsg.), Schriften (Bd. IV, S. 25-43). Frankfurt am Main: Suhrkamp.

Foucault, M. (2005b). Subjekt und Macht. In M. Foucault (Hrsg.), Schriften (Bd. IV, S. 269-294). Frankfurt am Main: Suhrkamp.

Foucault, M. (2006a). Sicherheit, Territorium, Bevölkerung. Geschichte der Gouvernementalität I. Frankfurt am Main: Suhrkamp.

Foucault, M. (2006b). Die Geburt der Biopolitik. Geschichte der Gouvernementalität II. Frankfurt am Main: Suhrkamp.

Füssel, M., \& Neu, T. (2010). Doing Discourse. Diskursiver Wandel aus praxeologischer Perspektive. In A. Landwehr (Hrsg.), Diskursiver Wandel (S. 213-253). Wiesbaden: VS.

Gertenbach, L. (2008). Geschichte, Zeit und sozialer Wandel: Konturen eines poststrukturalistischen Geschichtsdenkens. In S. Moebius \& A. Reckwitz (Hrsg.), Poststrukturalistische Sozialwissenschaften. Frankfurt am Main: Suhrkamp.

Hillebrandt, F. (2015). Was ist der Gegenstand einer Soziologie der Praxis? In F. Schäfer, A. Daniel \& F. Hillebrandt (Hrsg.), Methoden einer Soziologie der Praxis (S. 15-36). Bielefeld: transcript. 
Hirschauer, S. (2004). Praktiken und ihre Körper. Über materielle Partizipanden des Tuns. In K. H. Hörning \& J. Reuter (Hrsg.), Doing culture. Neue Positionen zum Verhältnis von Kultur und sozialer Praxis (S. 73-91). Bielefeld: transcript.

Keller, R. (2011). Wissenssoziologische Diskursanalyse. In R. Keller, A. Hirseland, W. Schneider \& W. Viehöver (Hrsg.), Handbuch sozialwissenschaftliche Diskursanalyse (3. Aufl. S. 114-146). Wiesbaden: VS.

Kessl, F. (2005). Soziale Arbeit als aktivierungspädagogischer Transmissionsriemen. In H.-J. Dahme \& N. Wohlfahrt (Hrsg.), Aktivierende Soziale Arbeit. Theorie - Handlungsfelder - Praxis (S. 30-43). Baltmannsweiler: Schneider.

Kessl, F. (2007). Wozu Studien zur Gouvernementalität in der Sozialen Arbeit? Von der Etablierung einer Forschungsperspektive. In R. Anhorn, F. Bettinger \& J. Stehr (Hrsg.), Foucaults Machtanalytik und Soziale Arbeit (S. 203-226). Wiesbaden: VS.

Kess1, F. (2011). Die Analyse von Rationalisierungspraktiken als Perspektive sozialpädagogischer Forschung. In B. Dollinger \& M. Schabdach (Hrsg.), Zugänge zur Geschichte der Sozialpädagogik und Sozialarbeit (S. 31-44). Siegen: Universitätsverlag.

Kessl, F., \& Krasmann, S. (2005). Sozialpolitische Programmierung. In F. Kessl, C. Reutlinger, S. Maurer \& O. Frey (Hrsg.), Handbuch Sozialraum (S. 227-245). Wiesbaden: VS.

Kessl, F., \& Otto, H.-U. (2008). Soziale Arbeit ohne Wohlfahrtsstaat? In F. Kessl \& H.-U. Otto (Hrsg.), Soziale Arbeit ohne Wohlfahrtsstaat? Zeitdiagnosen, Problematisierungen und Perspektiven (S. 7-21). Weinheim München: Juventa.

Kessl, F., \& Otto, H.-U. (2012). Soziale Arbeit. In G. Albrecht \& A. Groenemeyer (Hrsg.), Handbuch soziale Probleme (2. Aufl.) (S. 1306-1331). Wiesbaden: VS.

Laclau, E. (2007). Ideologie und Post-Marxismus. In M. Nonhoff (Hrsg.), Diskurs, radikale Demokratie, Hegemonie. Zum politischen Denken von Ernesto Laclau und Chantal Mouffe (S. 25-39). Bielefeld: transcript.

Langer, A. (2008). Disziplinieren und entspannen. Körper in der Schule - eine diskursanalytische Ethnographie. Bielefeld: transcript.

Langer, A., \& Wrana, D. (2010). Diskursforschung und Diskursanalyse. In B. Friebertshäuser, A. Langer \& A. Prengel (Hrsg.), Handbuch Qualitative Forschungsmethoden in der Erziehungswissenschaft (3. Aufl.) (S. 335-350). Weinheim München: Juventa.

Latour, B. (2007). Eine neue Soziologie für eine neue Gesellschaft. Einführung in die Akteur-NetzwerkTheorie. Frankfurt am Main: Suhrkamp.

Lemke, T. (2011). Eine Kritik der politischen Vernunft: Foucaults Analyse der modernen Gouvernementalität. Berlin: Argument.

Lessenich, S. (2008). Die Neuerfindung des Sozialen. Der Sozialstaat im flexiblen Kapitalismus. Bielefeld: transcript.

Lessenich, S. (2012). Theorien des Sozialstaats zur Einführung. Hamburg: Junius.

Macghilchrist, F., Ott, M., \& Langer, A. (2014). Der praktische Vollzug von „Bologna“. Eine ethnographische Diskursanalyse. In J. Angermüller, M. Nonhoff, E. Herschinger, F. Macgilchrist, M. Reisigl \& J. Wedl (Hrsg.), Diskursforschung. Ein interdisziplinäres Handbuch. 2 Bd (S. 37-57). Bielefeld: transcript.

Meissner, H. (2010). Jenseits des autonomen Subjekts: zur gesellschaftlichen Konstitution von Handlungsfähigkeit im Anschluss an Butler, Foucault und Marx. Bielefeld: transcript.

Miller, P., \& Rose, N. (1994). Das ökonomische Leben regieren. In R. Schwarz (Hrsg.), Zur Genealogie der Regulation. Anschlüsse an Michel Foucault (S. 54-108). Mainz: Decaton.

Moebius, S. (2008). Macht und Hegemonie: Grundrisse einer poststrukturalistischen Analytik der Macht. In S. Moebius \& A. Reckwitz (Hrsg.), Poststrukturalistische Sozialwissenschaften. Frankfurt am Main: Suhrkamp.

Moebius, S., \& Reckwitz, A. (2008). Poststrukturalistische Sozialwissenschaften. Frankfurt am Main: Suhrkamp.

Ott, M., \& Wrana, D. (2010). Gouvernementalität diskursiver Praktiken. Zur Methodologie der Analyse von Machtverhältnissen am Beispiel einer Maßnahme zur Aktivierung von Erwerbslosen. In J. Angermüller \& S. van Dyk (Hrsg.), Diskursanalyse meets Gouvernementalitätsforschung. Perspektiven auf das Verhältnis von Subjekt, Sprache, Macht und Wissen (S. 155-181). Frankfurt am Main: Campus.

Ott, M., Langer, A., \& Rabenstein, K. (2012). Integrative Forschungsstrategien - Ethnographie und Diskursanalyse verbinden. In B. Friebertshäuser, H. Kelle, H. Boller, S. Bollig, C. Huf \& A. Langer (Hrsg.), Feld und Theorie. Herausforderungen erziehungswissenschaftlicher Ethnographie (S. 169-184). Opladen: Budrich. 
Ott, M., Langer, A., \& Macgilchrist, F. (2014). Stichwort: Diskursanalyse, ethnographische. In D. Wrana, A. Ziem, M. Reisigl, M. Nonhoff \& J. Angermüller (Hrsg.), DiskursNetz. Wörterbuch der interdisziplinären Diskursforschung (S. 89-90). Berlin: Suhrkamp.

Poulantzas, N. (2002). Staatstheorie. Politischer Überbau, Ideologie, autoritärer Etatismus. Hamburg: VSA.

Rammert, W. (2007). Technik - Handeln - Wissen. Zu einer pragmatistischen Technik- und Sozialtheorie. Wiesbaden: VS.

Rammert, W., \& Schulz-Schaeffer, I. (2002). Technik und Handeln. Wenn soziales Handeln sich auf menschliches Verhalten und technische Artefakte verteilt. In W. Rammert \& I. Schulz-Schaeffer (Hrsg.), Können Maschinen handeln? Soziologische Beiträge zum Verhältnis von Mensch und Technik (S. 11-64). Frankfurt am Main: Campus.

Reckwitz, A. (2003). Grundelemente einer Theorie sozialer Praktiken. Eine sozialtheoretische Perspektive. ZfS, 32, 282-301.

Reckwitz, A. (2008). Praktiken und Diskurse: eine sozialtheoretische und methodologische Relation. In H. Kalthoff, S. Hirschauer \& G. Lindemann (Hrsg.), Theoretische Empirie: zur Relevanz qualitativer Forschung (S. 188-209). Frankfurt am Main: Suhrkamp.

Scharl, K., \& Wrana, D. (2014). Wahrheitspoliltik(en) zu „Bologna“ in einer Podiumsdiskussion. Eine praxeologisch-poststrukturalistische Figurationsanalyse. In J. Angermüller, E. Herschinger \& F. Macgilchrist (Hrsg.), Diskursforschung. Ein interdisziplinäres Handbuch. 2 Bd (S. 350-378). Bielefeld: transcript.

Schauz, D. (2010). Diskursiver Wandel am Beispiel der Disziplinarmacht. Geschichtstheoretische Implikationen der Dispositivanalyse. In A. Landwehr (Hrsg.), Diskursiver Wandel. Wiesbaden: VS.

West, C., \& Zimmerman, D.H. (1987). Doing gender. Gender and Society, 1, 125-151.

Wrana, D. (2006). Das Subjekt schreiben. Reflexive Praktiken und Subjektivierung in der Weiterbildung eine Diskursanalyse. Baltmannsweiler: Schneider.

Wrana, D. (2012a). Diesseits von Diskursen und Praktiken. Methodologische Bemerkungen zu einem Verhältnis. In B. Friebertshäuser, H. Kelle, H. Boller, S. Bollig, C. Huf \& A. Langer (Hrsg.), Feld und Theorie. Herausforderungen erziehungswissenschaftlicher Ethnographie (S. 185-200). Opladen: Barbara Budrich.

Wrana, D. (2012b). Theoretische und methodologische Grundlagen der Analyse diskursiver Praktiken. In D. Wrana \& C. Maier-Reinhard (Hrsg.), Professionalisierung in Lernberatungsgesprächen. Theoretische Grundlegungen und Empirische Untersuchungen (S. 195-214). Opladen: Barbara Budrich.

Ziegler, H. (2008). Sozialpädagogik nach dem Neo-Liberalismus: Skizzen einer post-sozialstaatlichen Formierung Sozialer Arbeit. In B. Bütow, K. A. Chassé \& R. Hirt (Hrsg.), Soziale Arbeit nach dem sozialpädagogischen Jahrhundert. Positionsbestimmungen Sozialer Arbeit im Post-Wohlfahrtsstaat (S. 159-176). Opladen: Budrich. 